\title{
Importance of Pancreatic Duct Stenting and Enteral Feeding in Treatment Algorithm of Pancreatic Fluid Collections
}

\author{
Seong-Hun Kim ${ }^{1,2,3}$ and Eun Ji Shin ${ }^{4}$ \\ ${ }^{1}$ Department of Internal Medicine, Jeonbuk National University Medical School and Hospital, Jeonju, Korea, ${ }^{2}$ Research Institute of \\ Clinical Medicine, Jeonbuk National University, Jeonju, Korea, ${ }^{3}$ Biomedical Research Institute, Jeonbuk National University Hospital, \\ Jeonju, Korea, ${ }^{4}$ Division of Gastroenterology and Hepatology, Johns Hopkins School of Medicine, Baltimore, MD, USA
}

See "Endoscopic Therapy for Pancreatic Fluid Collections: A Definitive Management Using a Dedicated Algorithm" by Ming Ming $\mathrm{Xu}$, Iman Andalib, Aleksey Novikov, et al., on page 355-360.

Despite advancements in the treatment of acute pancreatitis, early treatment remains a challenge, as mortality can reach up to $30 \%$ in severe cases. ${ }^{1}$ The Atlanta Classification for assessing the severity of acute pancreatitis was revised by international consensus in 2012. ${ }^{2}$ A critical aspect of pancreatitis treatment is the appropriate management of pancreatic fluid collections (PFCs). Acute pancreatitis-associated PFCs are classified as acute peripancreatic fluid collection, pancreatic pseudocyst (PP), acute necrotic collection (ANC), and walled-off necrosis (WON), based on the duration and presence or absence of necrosis. ${ }^{2}$ Particularly, disconnected pancreatic duct syndrome (DPDS) is linked with complications accompanied by ANC and WON, and characterized by stricture and discontinuity of pancreatic duct (PD) in PD injury. However, the guidelines for management of DPDS are not well established. ${ }^{3}$ The 2013 the International Association of Pancreatology (IAP)/the American Pancreatic Association (APA) evidence-based guidelines suggested an intervention (either radiological, endoscopic, or surgical) if pain or obstruction is present in DPDS with sterile

Received: April 24, 2020 Revised: May 13, 2020

Accepted: May 15, 2020

Correspondence: Eun Ji Shin

Division of Gastroenterology and Hepatology, Johns Hopkins School of Medicine Sheikh Zayed Tower, Suite 7125H, Baltimore, MD 21287, USA

Tel: +1-410-614-0950, Fax: +1-443-683-8333, E-mail: eshin3@jhmi.edu

ORCID: https://orcid.org/0000-0003-0624-8149

cc This is an Open Access article distributed under the terms of the Creative Commons Attribution Non-Commercial License (http://creativecommons.org/ licenses/by-nc/3.0) which permits unrestricted non-commercial use, distribution, and reproduction in any medium, provided the original work is properly cited. necrotizing pancreatitis, but there was no recommendation for the optimal timing of PD stenting. ${ }^{1}$ However, the 2013 American College of Gastroenterology guideline, ${ }^{4}$ and the 2015 revised Japanese guideline $e^{5}$ mentioned the role of $\mathrm{PD}$ stent for preventing post endoscopic retrograde cholangiopancreatography (ERCP) pancreatitis. In the 2018 American Gastroenterological Association Institute guideline, ${ }^{6}$ the indication for ERCP only deals with acute biliary pancreatitis with no guidance on the timing of PD stenting.

A study conducted by $\mathrm{Xu}$ et al. demonstrated the importance of PD stenting in acute pancreatitis-associated PFCs. ${ }^{7}$ The study included a total of 108 patients treated for acute pancreatitis-associated PFCs between September 2011 and October 2017. ERCP was performed on 74 patients (68.5\%) with suspected PD injury by magnetic resonance cholangiopancreatography (MRCP) before PFCs drainage. PD stent was placed in 53 (72\%) of 74 patients for treatment and in 19 (26\%) patients for prophylaxis. In their case, PD cannulation failed in 2 (3\%) patients. Using multivariate logistic regression analysis the authors reported that, "after adjusting for the presence of PD leak, PFC type, necrosectomy, and enteral feeding, PD stenting was almost five times (odds ratio [OR], 4.8; 95\% confidence interval [CI], 1.5-12.2) more likely to achieve resolution within 90 days $(p=0.0069))^{\prime 7}$. As 53 (49\%) out of the 108 patients received PD stenting for therapeutic purposes and the incidence of PD injury was found to be similar to that in previous studies on acute pancreatitis-associated PFCs, PD stenting can be considered as one of the important treatment strategies for acute pancreatitis-associated PFCs. ${ }^{3}$ 
Furthermore, two aspects regarding PD stenting results obtained in the above study need to be clarified. Firstly, one can mistakenly conclude that the 74 patients treated with ERCP (68.5\%) exhibited better PFCs treatment outcomes than the 34 patients who did not undergo ERCP (31\%), because the authors explicitly stated that the improvement of PFCs is better in patients with PD stenting than in those without it. However, one must remember that the 34 patients who did not undergo ERCP did not show suspected PD injury on MRCP, and thus would not exhibit additional benefits upon PD stenting. Therefore, for improved clarity, it would have been useful if the results were presented separately for the groups showing suspected PD injury with and without PD stenting. Secondly, the timings of PFCs drainage and PD stenting have not been clearly defined. The algorithm of Xu et al. ${ }^{7}$ was used to perform ERCP before PFCs drainage. Previous studies have not verified whether PD stenting should be performed before or after the PFCs drainage. In the presence of PFCs, intraluminal bulging and adhesion in the stomach and duodenum by extrinsic compression can hinder ERCP procedure, and thus may pose a risk of ERCP pancreatitis. Even in the study conducted by Xu et al., 2 (3\%) of the 74 patients who underwent ERCP with suspected PD injury on MRCP showed failed PD cannulation. ${ }^{7}$ Therefore, it is important to conduct comparative studies in the future on PD stenting before and after drainage procedure of the PFCs, if PD injury is suspected in acute pancreatitis-associated PFCs in order to determine the optimal timing of ERCP.

Another significant finding of the above-mentioned study is the importance of early enteral feeding in acute pancreatitis-associated PFCs. The authors used enteral feeding tubes in $65(60 \%)$ of the 108 patients. However, the rate of enteral feeding tube insertion is higher in WON $(45 / 52,86.5 \%)$ than poorly organized fluid collection $(6 / 13,46 \%)$ and PP (14/43, $33 \%$ ), which were poorly organized indicative of severe disease requiring more frequent enteral feeding. Unlike PD stenting, he importance of enteral feeding is well summarized in previous studies and guidelines. ${ }^{1,4-6,8}$ The authors reported that "after adjusting for the presence of PD leak, PFC type, necrosectomy, and PD stenting, enteral feeding was 3.4 times (OR, 3.4; 95\% CI, 1-10.9) more likely to achieve resolution within 60 days $(p=0.0421)$ ". Therefore, it is noteworthy that they confirmed the importance of enteral feeding, as is also included in the existing guidelines.

In previous studies, nasojejunal feeding (NJ) has been recommended over nasogastric feeding (NG), but the superiority of either method is still debatable. ${ }^{8}$ The therapeutic effects of enteral feeding within 24 hours and after 72 hours were similar. ${ }^{8}$ Of the 65 patients who received enteral feeding in this study, 5 (8\%) were subjected to NJ and 60 (92\%) were sub- jected to percutaneous gastrojejunostomy (PGJ). ${ }^{7}$ The feeding tube can be inserted by interventional radiology, endoscopy, or under electromagnetic guidance. This study mostly used the PGJ, and not NJ or NG, which is also recommended in major guidelines. PGJ reduces nasal complications and patient discomfort associated with long-term NJ or NG tube insertion, but dehiscence or leakage may develop at the site of gastrostomy and form a fistulous tract. ${ }^{8}$ The study conducted by Xu et al. also had this limitation, as it did not segregate the severity of acute pancreatitis. ${ }^{7}$ Enteral feeding varies depending on the severity of acute pancreatitis, and this might have affected their interpretation of results.

In conclusion, the implementation of endoscopic ultrasonography-guided drainage and endoscopic necrosectomy with early enteral feeding in acute pancreatitis-associated PFCs is a well-known strategy. Xu et al. demonstrated the importance of PD stenting by assessing the presence of PD injury in acute pancreatitis-associated PFCs. ${ }^{7}$ However, prospective studies to investigate the optimal timing of PD stenting and the effects of PD stenting in PFCs treatment algorithm are necessary.

\section{Conflicts of Interest}

Eun Ji Shin is Consultant for Boston Scientific and Medtronic. The other author has no financial conflicts of interest.

ORCID

Seong-Hun Kim: https://orcid.org/0000-0002-7592-8060

\section{REFERENCES}

1. Working Group IAP/APA Acute Pancreatitis Guidelines. IAP/APA evidence-based guidelines for the management of acute pancreatitis. Pancreatology 2013;13(4 Suppl 2):e1-e15.

2. Banks PA, Bollen TL, Dervenis C, et al. Classification of acute pancreatitis--2012: revision of the Atlanta classification and definitions by international consensus. Gut 2013;62:102-111.

3. Bang JY, Wilcox CM, Navaneethan U, et al. Impact of disconnected pancreatic duct syndrome on the endoscopic management of pancreatic fluid collections. Ann Surg 2018;267:561-568.

4. Tenner S, Baillie J, DeWitt J, Vege SS. American College of Gastroenterology guideline: management of acute pancreatitis. Am J Gastroenterol 2013;108:1400-1415; 1416.

5. Yokoe M, Takada T, Mayumi T, et al. Japanese guidelines for the management of acute pancreatitis: Japanese guidelines 2015. J Hepatobiliary Pancreat Sci 2015;22:405-432.

6. Crockett SD, Wani S, Gardner TB, Falck-Ytter Y, Barkun AN. American gastroenterological association institute guideline on initial management of acute pancreatitis. Gastroenterology 2018;154:1096-1101.

7. Xu MM, Andalib I, Novikov A, et al. Endoscopic therapy for pancreatic fluid collections: a definitive management using a dedicated algorithm. Clin Endosc 2020;53:355-360.

8. Trikudanathan G, Wolbrink DRJ, van Santvoort HC, Mallery S, Freeman M, Besselink MG. Current concepts in severe acute and necrotizing pancreatitis: an evidence-based approach. Gastroenterology 2019;156:1994-2007.e3. 